\title{
The growth of regular functions on algebraic sets
}

\author{
by A. Strzeboński (Kraków)
}

\begin{abstract}
We are concerned with the set of all growth exponents of regular functions on an algebraic subset $V$ of $\mathbb{C}^{n}$. We show that its elements form an increasing sequence of rational numbers and we study the dependence of its structure on the geometric properties of $V$.
\end{abstract}

1. Introduction. Let $V \subset \mathbb{C}^{N}$ be an algebraic set of positive dimension and let $f \in \mathbb{C}[V]$. Then, by the definition of $\mathbb{C}[V], f$ is the restriction to $V$ of a polynomial $F \in \mathbb{C}\left[X_{1}, \ldots, X_{N}\right]$ and so there exist two non-negative constants $A, B$ such that

$$
|f(z)| \leq A(1+|z|)^{B} \quad \text { for } z \in V,
$$

where $|\cdot|$ is a norm in $\mathbb{C}^{N}$ and $B \leq \operatorname{deg} F$. For simplicity, throughout the paper we will use the norm

$$
\left|\left(z_{1}, \ldots, z_{N}\right)\right|=\max _{1 \leq i \leq N}\left|z_{i}\right|
$$

Define

$$
\begin{aligned}
M(V, f) & :=\left\{B \geq 0: \exists A \geq O \text { such that }|f(z)| \leq A(1+|z|)^{B} \text { for } z \in V\right\}, \\
B(V, f) & :=\inf M(V, f), \\
B_{V} & :=\{B(V, f): f \in \mathbb{C}[V]\} .
\end{aligned}
$$

We will call $B(V, f)$ the growth exponent of $f$. The aim of this paper is to study the dependence of the structure of the set $B_{V}$ of growth exponents on the geometric properties of $V$.

In Section 2 it is shown (Theorem 2.1) that $M(V, f) \neq \emptyset, B(V, f) \in$ $M(V, f)$ and

$$
\mathbb{N} \subset B_{V} \subset\{p / q: p, q \in \mathbb{N},(p, q)=1,1 \leq q \leq d\},
$$

where $d$ is the maximum degree of the irreducible components of $V$ and $(p, q)$ denotes the greatest common divisor of $p$ and $q$.

1991 Mathematics Subject Classification: 32H30, 14N99, 32J25. 
Examples 3.1-3.4 show that all combinations of equalities and strict inclusions are possible in this theorem. The fact that $n a \in B_{V}$ whenever $a \in B_{V}$ and $n \in \mathbb{N}$ allows us to call $G \subset B_{V}$ a generating set of $B_{V}$ if for each $a \in B_{V}$ there exist $g \in G, n \in \mathbb{N}$ such that $a=n g$. The set $B_{V}$ defined in Example 3.5 is not closed under addition and has no one-element generating set. In Examples 3.6, 3.7 the sets $B_{V}$ are not generated by any finite set.

In Section 4 we show that neither the smallest number of generators nor the number of denominators of irreducible ratios belonging to $B_{V}$ are invariants of biregular mappings of $\mathbb{C}^{N}$. However, if $V$ has a one-dimensional polynomial parametrization we can calculate $B_{V}$. In particular, if $V$ is biregular with $\mathbb{C}$ then $B_{V}$ is generated by one element (Proposition 4.2).

The theorems of Section 5 give us a better characterization of $B_{V}$. If $V$ is a curve in $\mathbb{C}^{N}$ then $B_{V}$ is contained in the set of ratios with denominators equal to the intersection multiplicities of the irreducible components of the analytic germs of the projective closure of $V$ at points at infinity with the hyperplane at infinity. If $V \subset \mathbb{C}^{N}$ is an algebraic set of pure dimension then there exist natural numbers $q_{1}, \ldots, q_{r}$ such that $q_{1}+\ldots+q_{r} \leq \operatorname{deg} V$, $B_{V} \subset\left\{m / q_{i}: i=1, \ldots r, m \in \mathbb{N}\right\}$. The proof of this fact bases on the following property: if $f \in \mathbb{C}[V]$ then $B(C, f \mid C)=B(V, f)$ for "almost all" algebraic curves $C \subset V$.

Section 6 deals with the case of a hypersurface $V$. We study the dependence of $B_{V}$ on the multiplicities of the irreducible factors of the leading form of the polynomial describing $V$. In particular, we show that if $B_{V}$ contains an irreducible ratio with denominator $k$, then the leading form of the polynomial describing $V$ is divisible by the $k$ th power of a homogeneous non-constant polynomial.

\section{The basic theorem}

THEOREM 2.1. If $V \subset \mathbb{C}^{N}$ is an algebraic set of positive dimension and if $f \in \mathbb{C}[V]$ then $M(V, f) \neq \emptyset$ and $B(V, f) \in M(V, f)$. Moreover, if d denotes the maximum degree of the irreducible components of $V$, then

$$
\mathbb{N} \subset B_{V} \subset D_{d}:=\{p / q: p, q \in \mathbb{N},(p, q)=1,1 \leq q \leq d\} .
$$

The theorem will be proved by means of a sequence of lemmas.

LEMMA 2.2. If $V \subset \mathbb{C}^{n+k}$ is an algebraic set of pure dimension $n>0$ and of degree $d$ and if $f \in \mathbb{C}[V]$, then $B(V, f) \in M(V, f)$ and $B_{V} \subset D_{d}$.

Proof. Let $f \in \mathbb{C}[V]$. By Sadullaev's theorem (see [2], VII, 7.1) we may assume that for some $C>0$

$$
V \subset\left\{(x, y) \in \mathbb{C}^{n} \times \mathbb{C}^{k}:|y| \leq C(1+|x|)\right\} .
$$


Let $\Phi: V \ni(x, y) \rightarrow(x, f(x, y)) \in \mathbb{C}^{n} \times \mathbb{C}$. Since $\Phi$ is a proper holomorphic mapping, $W:=\Phi(V)$ is an analytic set of pure dimension $n$, by Remmert's theorem.

The projection $\pi \mid W: W \ni(x, t) \rightarrow x \in \mathbb{C}^{n}$ is proper, hence there exist a proper analytic subset $S$ of $\mathbb{C}^{n}$ and a natural number $s$ such that $\#(\pi \mid W)^{-1}(x)=s$ for all $x \in \mathbb{C}^{n} \backslash S$, and there exist holomorphic functions $\sigma_{1}, \ldots, \sigma_{s}: \mathbb{C}^{n} \rightarrow \mathbb{C}$ such that

$$
W=\left\{(x, t) \in \mathbb{C}^{n} \times \mathbb{C}: t^{s}+\sigma_{1}(x) t^{s-1}+\ldots+\sigma_{s}(x)=0\right\}
$$

(see e.g. [4], p. 71, Lemma 1).

Clearly, $\#(\pi \mid W)^{-1}(x) \leq \operatorname{deg} V=d$ for $x \in \mathbb{C}^{n}$, and so $s \leq d$. By the Vieta formulae we obtain

$$
\left|\sigma_{i}(x)\right| \leq\left(\begin{array}{c}
s \\
i
\end{array}\right) \max \left\{|t|^{i}:(x, t) \in W\right\}=\left(\begin{array}{c}
s \\
i
\end{array}\right) \max \left\{|f(x, y)|^{i}:(x, y) \in V\right\} .
$$

Moreover, if $f=F \mid V, F \in \mathbb{C}\left[X_{1}, \ldots, X_{n+k}\right]$ then for some $A>0$

$$
\begin{aligned}
|f(x, y)| & \leq A(1+|(x, y)|)^{\operatorname{deg} F} \leq A(1+|x|+C(1+|x|))^{\operatorname{deg} F} \\
& =(C+1)^{\operatorname{deg} F} A(1+|x|)^{\operatorname{deg} F} .
\end{aligned}
$$

Hence, by the Liouville theorem, $\sigma_{i}(x)$ is a polynomial (for $i=1, \ldots, s$ ). Thus $W$ is algebraic.

Now, it suffices to prove

LEMMA 2.3. Let $W=\left\{(x, t) \in \mathbb{C}^{n} \times \mathbb{C}: t^{s}+\sigma_{1}(x) t^{s-1}+\ldots+\sigma_{s}(x)=0\right\}$, where $\sigma_{i}$ is a polynomial of degree $p_{i}$ for $i=1, \ldots, s$. If $B:=$ $\max _{1 \leq i \leq s}\left\{p_{i} / i\right\}, M(W):=\{D \geq 0: \exists A \geq 0$ such that $W \subset\{(x, t)$ : $\left.\left.|t| \leq \bar{A}(1+|x|)^{D}\right\}\right\}$, then $B \in M(W)$ and $B=\min M(W)$.

Indeed, from (1) and the definition of $W$ it follows that $M(V, f)=$ $M(W)$. Thus, since $s \leq d$, Lemma 2.3 implies Lemma 2.2. To prove Lemma 2.3, choose $A_{0}$ such that $\left|\sigma_{i}(x)\right| \leq A_{0}(1+|x|)^{p_{i}}$ for $i=1, \ldots, s$. Set $A:=\max \left\{s A_{0}, 1\right\}$. Suppose that there exist $(x, t) \in W$ such that $|t|>A(1+|x|)^{B}$. Since $t^{s}=-\sigma_{1}(x) t^{s-1}+\ldots-\sigma_{s}(x)$ for $(x, t) \in W$, we have

$$
\begin{aligned}
|t| & =\left|\sigma_{i}(x)+\frac{\sigma_{2}(x)}{t}+\ldots+\frac{\sigma_{s}(x)}{t^{s-1}}\right| \leq \sum_{i=1}^{s} \frac{\left|\sigma_{i}(x)\right|}{|t|^{i-1}} \\
& \leq \frac{\sum_{i=1}^{s} A_{0}(1+|x|)^{p_{i}}}{A^{i-1}(1+|x|)^{B(i-1)}}=\sum_{i=1}^{s} \frac{A_{0}}{A^{i-1}}(1+|x|)^{p_{i}-B(i-1)} .
\end{aligned}
$$

Since $p_{i}-B(i-1) \leq p_{i}-\left(p_{i} / i\right)(i-1)=p_{i} / i \leq B$, we obtain

$$
|t| \leq\left(\sum_{i=1}^{s} A_{0} / A^{i-1}\right)(1+|x|)^{B}
$$


which contradicts our assumption. Hence $W \subset\left\{(x, t):|t| \leq A(1+|x|)^{B}\right\}$, and so $B \in M(W)$.

Suppose now that there exists $D \in M(W)$ such that $D<B$. Then there exist $A>0$ and $1 \leq i \leq s$ such that $D<p_{i} / i$ and $|t| \leq A(1+|x|)^{D}$ for $(x, t) \in W$. By the Vieta formulae

$$
\left|\sigma_{i}(x)\right| \leq\left(\begin{array}{c}
s \\
i
\end{array}\right) A^{i}(1+|x|)^{i D},
$$

and so $\operatorname{deg} \sigma_{i} \leq D i<p_{i}$. This contradiction completes the proof of Lemma 2.3.

As a simple consequence of Lemma 2.2 we obtain

Lemma 2.4. If $V=\bigcup_{j=1}^{s} V_{j}$ is the decomposition of an algebraic set $V \subset \mathbb{C}^{N}$ into irreducible components and if $f \in \mathbb{C}[V]$, then

$$
B(V, f)=\max _{1 \leq j \leq s} B\left(V_{j}, f \mid V_{j}\right) \in M(V, f) .
$$

To prove Theorem 2.1 it remains to show $\mathbb{N} \subset B_{V}$. By Sadullaev's theorem we can assume that, for some $C>0$,

$$
V \subset\left\{(x, y) \in \mathbb{C}^{n} \times \mathbb{C}^{N-n}:|y|<C(1+|x|)\right\},
$$

where $n=\operatorname{dim} V$. Since the projection $\pi \mid V: V \ni(x, y) \rightarrow x \in \mathbb{C}^{n}$ is proper, by the Remmert theorem, $\pi(V)=\mathbb{C}^{n}$. Now, it is easy to check that $B\left(V, X_{1}^{k}\right)=k$ for $k \in \mathbb{N}$, where $x=\left(x_{1}, \ldots, x_{n}\right)$.

3. Examples. Under the assumptions of Theorem 2.1 we have $\mathbb{N} \subset$ $B_{V} \subset D_{d}$. The following examples show that all combinations of equalities and strict inclusions are possible here.

Example 3.1. If $V$ is a linear subspace of $\mathbb{C}^{N}$, then $\mathbb{N}=B_{V}=D_{d}$.

Example 3.2. Let $V=\left\{(x, y) \in \mathbb{C}^{2}: x y=1\right\}$. Then $\mathbb{N}=B_{V} \varsubsetneqq D_{d}=$ $\{n / 2: n \in \mathbb{N}\}$.

Example 3.3. Let $V=\left\{(x, y) \in \mathbb{C}^{2}: y-x^{2}=0\right\}$. Then $\mathbb{N} \varsubsetneqq B_{V}=$ $D_{d}=\{n / 2: n \in \mathbb{N}\}$.

ExAmple 3.4. Let $V=\left\{(x, y) \in \mathbb{C}^{2}: y-x^{n}=0\right\}, n>2$. If $|(x, y)| \geq 1$, $(x, y) \in V$ then $|(x, y)|=|y|$. For $f \in \mathbb{C}[V]$,

$$
f(x, y)=\sum_{\text {finite }} a_{i, j} x^{i} y^{j}=\sum a_{i, j} \varepsilon_{n}^{i} y^{j+i / n},
$$

where $a_{i, j} \in \mathbb{C}, \varepsilon_{n}^{n}=1$. Hence $B_{V}=\{k / n: k \in \mathbb{N}\}$. As $d=\operatorname{deg} V=n$ we have $\mathbb{N} \varsubsetneqq B_{V} \varsubsetneqq D_{d}$.

Example 3.5. Let $k, n \in \mathbb{N}_{+}, V=\left\{(x, y) \in \mathbb{C}^{2}: x^{n} y^{k}=1\right\}$. Then $B_{V}=\{i s / k, j s / n: i, j \in \mathbb{N}, s:=(k, n)\}$. Choosing for instance $k=2$, 
$n=3$ we obtain the set $B_{V}$ not closed under addition and having no oneelement generating set.

Example 3.6. Let $k, n \in \mathbb{N}_{+}$and let $n \leq k$. For $V=\left\{(x, y) \in \mathbb{C}^{2}\right.$ : $\left.x^{n}-y^{k}=0\right\}$ we have $B_{V}=\{i n / k+j: i, j \in \mathbb{N}\}$. In particular, for $n=2$ and $k=3 B_{V}=\{0,2 / 3,1,4 / 3,5 / 3,2, \ldots\}=\{n / 3: n \in \mathbb{N} \backslash\{1\}\}$.

Example 3.7. If $V=\left\{(x, y) \in \mathbb{C}^{2}:\left(y^{3}-1\right) x^{2}-1=0\right\}$, then $B_{V}=$ $\{0,1,3 / 2,2,5 / 2, \ldots\}=\{n / 2: n \in \mathbb{N} \backslash\{1\}\}$.

\section{The growth exponents on biregular sets}

EXAMPLE 4.1. Neither the smallest number of generators of $B_{V}$ nor the number of denominators of irreducible ratios belonging to $B_{V}$ are invariants of biregular mappings of $\mathbb{C}^{N}$. For instance the biregular mapping $\Phi: \mathbb{C}^{2} \ni$ $(x, y) \rightarrow\left(x-y^{3}, y\right) \in \mathbb{C}^{2}$ maps $V=\left\{(x, y) \in \mathbb{C}^{2}: x y^{2}-1=0\right\}$ onto $W=\left\{(w, z) \in \mathbb{C}^{2}:\left(w+z^{3}\right) z^{2}-1=0\right\}$ and $B_{V}=\{i / 2: i \in \mathbb{N}\}, B_{W}=$ $\{i / 2, j / 3: i, j \in \mathbb{N}\}$.

Proposition 4.2. Let $f=\left(f_{1}, \ldots, f_{n}\right): \mathbb{C} \rightarrow \mathbb{C}^{n}$ be a polynomial mapping and let $V=f(\mathbb{C})$. If $p:=\max _{1 \leq i \leq n} \operatorname{deg} f_{i}$ then

$$
B_{V}=\{i / p: \exists h \in \mathbb{C}[V] \text { such that } \operatorname{deg}(h \circ f)=i\} .
$$

In particular, if $V$ is biregular with $\mathbb{C}$ then $B_{V}=\{i / p: i \in \mathbb{N}\}$.

The proof of this proposition bases on the following

Lemma 4.3. If $a, b \in \mathbb{C}[t], \operatorname{deg} a=r, \operatorname{deg} b=s$ then there exists $A>0$ such that $|a(t)| \leq A(1+|b(t)|)^{r / s}, t \in \mathbb{C}$.

Since $\lim _{t \rightarrow \infty}\left|a(t)^{s} / b(t)^{r}\right|=M \in(0, \infty)$, there exists $R$ such that $\left|a(t)^{s} / b(t)^{r}\right|<2 M$ for $|t|>R$, and so

$$
A:=\max \left\{(2 M)^{1 / s}, \sup _{|t| \leq R}|a(t)|\right\}
$$

is the required constant.

To prove Proposition 4.2 choose $i$ such that $\operatorname{deg} f_{i}=p$ and let $h \in \mathbb{C}[V]$. If $r:=\operatorname{deg}(h \circ f)$ then, by Lemma 4.3, there exists $A>0$ such that

$$
|h(x)|=|h \circ f(t)| \leq A\left(1+\left|f_{i}(t)\right|\right)^{r / p} \leq A(1+|x|)^{r / p},
$$

where $x=f(t), t \in \mathbb{C}$. Again by Lemma 4.3 , there exists $A^{\prime}$ such that

$$
\left|f_{j}(t)\right| \leq A^{\prime}(1+|h \circ f(t)|)^{p / r} \quad \text { for } j=1, \ldots, n .
$$

Since $|x|=\max _{1 \leq j \leq n}\left|x_{j}\right|$, putting $A^{\prime \prime}:=A^{\prime-r / p}$ we obtain

$$
A^{\prime \prime}|x|^{r / p}-1 \leq|h(x)| \leq A(1+|x|)^{r / p} .
$$

Hence $B(V, h)=r / p$. 
5. A sharpened version of the basic theorem. Let $V \subset \mathbb{C}^{n} \subset \mathbb{P}^{n}$ be an affine algebraic set, let $\bar{V}$ denote its projective closure and let $H_{\infty}:=$ $\mathbb{P}^{n} \backslash \mathbb{C}^{n}$. For analytic subsets $W, Z$ of an open neighbourhood of $a \in \mathbb{P}^{n}$ let $i(W \cdot Z, a)$ denote the intersection multiplicity of $W$ and $Z$ at $a$ (in the sense of [1], see also [5]).

THEOREM 5.1. Let $V \subset \mathbb{C}^{n}$ be an algebraic set of pure dimension 1 . Let $\bar{V} \cap H_{\infty}=\left\{a_{1}, \ldots, a_{r}\right\}$ and let, for $i=1, \ldots, r, \bar{V}_{a_{i}}=A_{i, 1} \cup \ldots \cup A_{i, s_{i}}$ be the decomposition of the germ $\bar{V}_{a_{i}}$ into irreducible analytic germs. If $q_{i, j}:=i\left(A_{i, j} \cdot H_{\infty}, a_{i}\right)$ for $i=1, \ldots, r, j=1, \ldots, s_{i}$, then

$$
B_{V} \subset\left\{m / q_{i, j}: m \in \mathbb{N}, i=1, \ldots, r, j=1, \ldots, s_{i}\right\} .
$$

Proof. For any $Z \subset \mathbb{C}^{n}$ and $f \in \mathbb{C}\left[X_{1}, \ldots, X_{n}\right]$ we set $B(Z, f):=\inf \left\{B \geq 0: \exists A>0\right.$ such that $|f(x)| \leq A(1+|x|)^{B}$ for $\left.x \in Z\right\}$. Now, since $B\left(Z_{1} \cup \ldots \cup Z_{t}, f\right)=\max _{1 \leq i \leq t} B\left(Z_{i}, f\right)$ and $B(K, f)=0$ for any compact set $K$, it suffices to prove the following:

(L) Each germ $A_{i, j}, i=1, \ldots, r, j=1, \ldots, s_{i}$, has a representative $\mathcal{A}_{i, j}$ such that for any $f \in \mathbb{C}\left[X_{1}, \ldots, X_{n}\right]$ and any open neighbourhood $U_{i}$ of $a_{i}$ we have

(1) $\quad B_{i, j}(f):=B\left(\mathcal{A}_{i, j} \cap U_{i} \cap \mathbb{C}^{n}, f\right)$ does not depend on the choice of $U_{i}$,

$$
B_{i, j}(f) \in\left\{m / q_{i, j}: m \in \mathbb{N}\right\} .
$$

Fix $i \in\{1, \ldots, r\}, j \in\left\{1, \ldots, s_{i}\right\}$. In suitable projective coordinates $\left\langle x_{0}, \ldots, x_{n}\right\rangle$ in $\mathbb{P}^{n}$ we have $H_{\infty}=\left\{x_{0}=0\right\}, a:=a_{i}=\langle 0, \ldots, 0,1\rangle$. Then $A:=A_{i, j}$ is an irreducible analytic germ at $a, \operatorname{dim} A=1, q:=i\left(A \cdot H_{\infty}, a\right)=$ $q_{i, j}$ and $A \cap H_{\infty}=\{a\}$. By the Puiseux theorem (see e.g. [2], II, 6.2, III, 4.4), there exist $p \in \mathbb{N}_{+}$, a holomorphic mapping $h:\{t \in \mathbb{C}:|t|<\delta\} \rightarrow$ $\mathbb{C}^{n-1}$ with $h(0)=0$ and a representative $W$ of the germ $A$ such that $W=$ $\left\{\left\langle t^{p}, h(t), 1\right\rangle:|t|<\delta\right\}$ and the mapping $\{|t|<\delta\} \ni t \rightarrow\left\langle t^{p}, h(t), 1\right\rangle \in W$ is homeomorphic. The mapping

$$
\pi \mid W: W \ni\left\langle t^{p}, h(t), 1\right\rangle \rightarrow t^{p} \in\left\{\left\langle x_{0}, 0, \ldots, 0,1\right\rangle:\left|x_{0}\right|<\delta^{p}\right\}
$$

is a $p$-sheeted branched covering, and so $p=i\left(A \cdot H_{\infty}, a\right)=q$. If we set $\mathcal{A}_{i, j}:=W$ then

$$
\mathcal{A}_{i, j} \cap \mathbb{C}^{n}=\left\{\left\langle 1, h(t) t^{-q}, t^{-q}\right\rangle:|t|<\delta\right\} .
$$

As $h(0)=0$ we may assume that $|h(t)|<1$. Since $|x|=\max _{1 \leq j \leq n}\left|x_{j}\right|$, we have $|x|=|t|^{-q}$ for $x=x(t)=\left(h(t) t^{-q}, t^{-q}\right) \in \mathcal{A}_{i, j} \cap \mathbb{C}^{n}$.

Fix $f \in \mathbb{C}\left[X_{1}, \ldots, X_{n}\right]$. Then, for $x=x(t) \in \mathcal{A}_{i, j} \cap \mathbb{C}^{n}, f(x)=f(x(t))=$ $\sum_{m=d}^{\infty} f_{m} t^{m}$, where $f_{m} \in \mathbb{C}, d \in \mathbb{Z}, f_{d} \neq 0$. Since

$$
\left|f(x(t)) / t^{d}\right|=\left|\sum_{m=0}^{\infty} f_{m+d} t^{m}\right| \underset{t \rightarrow 0}{\longrightarrow}\left|f_{d}\right| \in(0, \infty),
$$


we have

$$
\frac{1}{2}\left|f_{d}\right||t|^{d} \leq|f(x(t))| \leq 2\left|f_{d}\right||t|^{d}, \quad \text { for }|t|<\delta^{\prime} .
$$

As $|t|=|x|^{-1 / q}$ we obtain

$$
\frac{1}{2}\left|f_{d}\right||x|^{-d / q} \leq|f(x)| \leq 2\left|f_{d}\right||x(t)|^{-d / q} .
$$

Now, since $B(K, f)=0$ for any bounded set $K$, and $B(W \cup Z, f)=$ $\max \{B(W, f), B(Z, f)\}$, it is easily seen that for any open neighbourhood $U_{i}$ of $a_{i}$

$$
B\left(\mathcal{A}_{i, j} \cap U_{i} \cap \mathbb{C}^{n}, f\right)=\max \{-d / q, 0\},
$$

which completes the proof of $(\mathrm{L})$.

The following theorem is a generalization of Theorem 5.1 for algebraic sets of any pure dimension. Since irreducible components of any algebraic set have pure dimension, we may apply this theorem to any algebraic set, by Lemma 2.4 .

THEOREM 5.2. If $V \subset \mathbb{C}^{N}$ is an algebraic set of pure dimension, then there exist natural numbers $q_{1}, \ldots, q_{r}$ such that $q_{1}+\ldots+q_{r} \leq \operatorname{deg} V$ and $B_{V} \subset\left\{m / q_{i}: i=1, \ldots, r, m \in \mathbb{N}\right\}$.

LEMmA 5.3. Let $V \subset\left\{(x, y) \in \mathbb{C}^{n} \times \mathbb{C}^{k}:|y| \leq C(1+|x|)\right\}$ be an algebraic set of pure dimension $n$, and let $f \in \mathbb{C}[V]$. For $x \in \mathbb{C}^{n}$ we put

$$
\begin{aligned}
l_{x}: & =\{a x: a \in \mathbb{C}\} \subset \mathbb{C}^{n}, \\
V_{x}: & =\pi^{-1}\left(l_{x}\right) \cap V, \text { where } \pi: \mathbb{C}^{n} \times \mathbb{C}^{k} \ni(u, w) \rightarrow u \in \mathbb{C}^{n}, \\
f_{x}: & =f \mid V_{x} .
\end{aligned}
$$

Then there exists an algebraic cone $Z \varsubsetneqq \mathbb{C}^{n}$ such that $B\left(V_{x}, f_{x}\right)=B(V, f)$ for $x \in \mathbb{C}^{n} \backslash Z$.

Proof. As in the proof of Lemma 2.2, we put

$$
\Phi: V \ni(x, y) \rightarrow(x, f(x, y)) \in \mathbb{C}^{n} \times \mathbb{C}, \quad W:=\Phi(V) .
$$

Then there exist $\sigma_{1}, \ldots, \sigma_{s} \in \mathbb{C}\left[X_{1}, \ldots, X_{n}\right]$ such that

$$
W=\left\{(x, t) \in \mathbb{C}^{n} \times \mathbb{C}: t^{s}+\sigma_{1}(x) t^{s-1}+\ldots+\sigma_{s}(x)=0\right\}
$$

and $B(V, f)=\max _{1 \leq i \leq s}\left\{p_{i} / i\right\}$, where $p_{i}=\operatorname{deg} \sigma_{i}$. If $\sigma_{i}=\sigma_{i, 0}+\ldots+\sigma_{i, p_{i}}$ is the decomposition of $\sigma_{i}$ into homogeneous forms, then

$$
\sigma_{i}^{x}(a):=\sigma_{i}(a x)=\sigma_{i, 0}+\ldots+\sigma_{i, p_{i}}(x) a^{p_{i}} \quad \text { for } a \in \mathbb{C} .
$$

Applying Lemma 2.3 to the set

$$
W_{x}=\Phi\left(V_{x}\right)=\left\{(a x, t): t^{s}+\sigma_{1}^{x}(a) t^{s-1}+\ldots+\sigma_{s}^{x}(a)=0\right\}
$$

we obtain

$$
B\left(V_{x}, f_{x}\right)=\max _{1 \leq i \leq s}\left\{\left(\operatorname{deg} \sigma_{i}^{x} / i\right\}\right.
$$


Then the set $Z \varsubsetneqq \mathbb{C}^{n}$ defined by

$$
Z:=\left\{x \in \mathbb{C}^{n}: \prod_{i=1}^{s} \sigma_{i, p_{i}}(x)=0\right\}
$$

is an algebraic cone and $B\left(V_{x}, f_{x}\right)=B(V, f)$ for $x \in \mathbb{C}^{n} \backslash Z$.

Proof of Theorem 5.2. Let $p_{1}, \ldots, p_{s}$ be all the different denominators of the irreducible ratios belonging to $B_{V}$ (see Theorem 2.1), and let $f_{1}, \ldots, f_{s} \in \mathbb{C}[V]$ be such that $B\left(V, f_{i}\right)=n_{i} / p_{i}$, where $\left(n_{i}, p_{i}\right)=1$ for $i=1, \ldots, s$. By Sadullaev's theorem, we can assume that $V \subset\{(x, y) \in$ $\left.\mathbb{C}^{n} \times \mathbb{C}^{N-n}:|y|<C(1+|x|)\right\}$, where $n=\operatorname{dim} V$. By Lemma 5.3 there exist algebraic cones $Z_{i} \varsubsetneqq \mathbb{C}^{n}$ such that

$$
B\left(V_{x_{i}},\left(f_{i}\right)_{x_{i}}\right)=n_{i} / p_{i} \quad \text { for } i=1, \ldots, s, x_{i} \in \mathbb{C} \backslash Z_{i} .
$$

Let $x \in \mathbb{C} \backslash\left(Z_{1} \cup \ldots \cup Z_{s}\right)$. The $V_{x}$ has pure dimension 1. By the Bézout theorem and Theorem 5.1,

$$
B_{V_{x}} \subset\left\{m / q_{i}: i=1, \ldots, r, m \in \mathbb{N}\right\},
$$

where $q_{1}+\ldots+q_{r} \leq \operatorname{deg} V_{x} \leq \operatorname{deg} V$. As $B\left(V_{x},\left(f_{i}\right)_{x}\right)=n_{i} / p_{i}, i=1, \ldots, s$, we have

$$
B_{V} \subset\left\{m / q_{i}: i=1, \ldots, r, m \in \mathbb{N}\right\}
$$

Corollary 5.4. For any algebraic set $V \subset \mathbb{C}^{N}$ of pure positive dimension there exists a curve $\Gamma \subset V$ such that $B_{V} \subset B_{\Gamma}$.

To prove the corollary we apply Lemma 5.3 and notice that a countable sum of proper algebraic cones is nowhere dense, by the Baire theorem.

6. The case of a hypersurface. Let $F \in \mathbb{C}\left[X_{1}, \ldots, X_{n}\right], n>0$. By a reduced decomposition of $F$ we mean a decomposition $F=p_{1}^{d_{1}} \ldots p_{l}^{d_{l}}$ such that

(1) $d_{1}<\ldots<d_{l}$

(2) $\left(p_{i}, p_{j}\right)=1$ for $i \neq j$,

(3) $p_{i}$ has no multiple factors for $i=1, \ldots, l$.

It is easily seen that each $F \in \mathbb{C}\left[X_{1}, \ldots, X_{n}\right]$ has a unique reduced decomposition.

TheOREM 6.1. If $V=\left\{x \in \mathbb{C}^{n}: F(x)=0\right\}$, where $F \in \mathbb{C}\left[X_{1}, \ldots, X_{n}\right]$, and if $F_{d}=p_{1}^{d_{1}} \ldots p_{l}^{d_{l}}$ is the reduced decomposition of the leading form of $F$, where $\operatorname{deg} p_{i}=r_{i}$, then there exist natural numbers $m_{i, j, k}$ for $i=1, \ldots, l$, $j=1, \ldots, r_{i}, k=1, \ldots, s_{i, j}$, such that $d_{i}=\sum_{k=1}^{s_{i, j}} m_{i, j, k}$ for $j=1, \ldots, r_{i}$ and

$$
B_{V} \subset\left\{t / m_{i, j, k}: t \in \mathbb{N}, i=1, \ldots, l, j=1, \ldots, r_{i}, k=1, \ldots, s_{i, j}\right\} .
$$


In particular, if $k$ is the maximum denominator of the irreducible ratios in $B_{V}$ then $F_{d}$ is divisible by the $k$-th power of a non-constant polynomial.

Lemma 6.2. If $V=\left\{(x, y) \in \mathbb{C}^{2}: F(x, y)=0\right\}, F \in \mathbb{C}[X, Y]$, then there exist $m_{1}, \ldots, m_{s} \in \mathbb{N}_{+}$and homogeneous linear polynomials $p_{1}, \ldots, p_{s} \in$ $\mathbb{C}[X, Y]$ such that $F_{d}=p_{1}^{m_{1}} \ldots p_{s}^{m_{s}}$ is the leading form of $F$ and $B_{V} \subset$ $\left\{n / m_{i}: n \in \mathbb{N}, i=1, \ldots, s\right\}\left(p_{1}, \ldots, p_{s}\right.$ need not be different $)$.

This lemma is a consequence of Theorem 5.1 for in the notation of that theorem

$$
\begin{aligned}
\sum_{j=1}^{s_{i}} i\left(A_{i, j} \cdot H_{\infty}, a_{i}\right) & =i\left(\bar{V}_{a_{i}} \cdot H_{\infty}, a_{i}\right) \\
& =\left\{\text { multiplicity of the factor } y_{i} X_{i}-x_{i} Y_{i} \text { in } F_{d}\right\},
\end{aligned}
$$

where $a_{i}=\left\langle 0, x_{i}, y_{i}\right\rangle$.

Proof of Theorem 6.1. We can assume, by changing linear coordinates if needed, that the coefficient at $X_{i}^{d}$ in $F_{d}$ is different from 0 for $i=1, \ldots, n$. Let $Y:=X_{n}, X^{\prime}:=\left(X_{1}, \ldots, X_{n-1}\right)$. Then, dividing $F$ by a non-zero constant if needed, we obtain

$$
F(X)=F\left(X^{\prime}, Y\right)=Y^{d}+a_{1}\left(X^{\prime}\right) Y^{d-1}+\ldots+a_{d}\left(X^{\prime}\right),
$$

where $a_{1}, \ldots, a_{d} \in \mathbb{C}\left[X^{\prime}\right], \operatorname{deg} a_{i} \leq i, \operatorname{deg} a_{d}=d$. From Lemma 2.3 we have

$$
V \subset\left\{\left(x^{\prime}, y\right) \in \mathbb{C}^{n-1} \times \mathbb{C}:|y| \leq C\left(1+\left|x^{\prime}\right|\right)\right\},
$$

where $C$ is a suitable constant.

Let $B_{V}=\left\{B\left(V, f_{m}\right): m \in \mathbb{N}\right\}$. Using the notation of Lemma 5.3, by that lemma, we obtain, for each $m \in \mathbb{N}$, an algebraic cone $Z_{m} \varsubsetneqq \mathbb{C}^{n-1}$ such that

The set

$$
B\left(V, f_{m}\right)=B\left(V_{x^{\prime}},\left(f_{m}\right)_{x^{\prime}}\right), \quad \text { for } x^{\prime} \in \mathbb{C}^{n-1} \backslash Z_{m} .
$$

$$
U:=\mathbb{C}^{n-1} \backslash \bigcup_{m=0}^{\infty} Z_{m}
$$

is of the second Baire category and $B_{V} \subset B_{V_{x^{\prime}}}$ for $x^{\prime} \in U$.

Let $x^{\prime} \in U$. Then $V_{x^{\prime}}=\left\{\left(a x^{\prime}, y\right) \in \mathbb{C}^{n}: F\left(a x^{\prime}, y\right)=0\right\}$. Write

$$
\begin{gathered}
F\left(x^{\prime}\right)(A, Y):=F\left(A x^{\prime}, Y\right), \quad F\left(x^{\prime}\right) \in \mathbb{C}[A, Y], \\
W\left(x^{\prime}\right):=\left\{(a, y) \in \mathbb{C}^{2}:\left(a x^{\prime}, y\right) \in V_{x^{\prime}}\right\}=\left\{(a, y) \in \mathbb{C}^{2}: F\left(x^{\prime}\right)(a, y)=0\right\}, \\
f\left(x^{\prime}\right)(a, y):=f\left(a x^{\prime}, y\right) \quad \text { for } f \in \mathbb{C}[V], f\left(x^{\prime}\right) \in \mathbb{C}\left[W\left(x^{\prime}\right)\right] .
\end{gathered}
$$

Since $\left|a x^{\prime}\right|=\left|x^{\prime}\right||a|, B\left(V_{x^{\prime}}, f_{x^{\prime}}\right)=B\left(W\left(x^{\prime}\right), f\left(x^{\prime}\right)\right)$ for $f \in \mathbb{C}[V]$. Hence $B_{V} \subset B_{W\left(x^{\prime}\right)}$. 
If $F_{d}\left(x^{\prime}\right)(A, Y):=F_{d}\left(A x^{\prime}, Y\right)$, then $F_{d}\left(x^{\prime}\right)$ is the leading form of $F\left(x^{\prime}\right)$. Since $F_{d}\left(x^{\prime}\right)$ is a homogeneous polynomial in two variables with the coefficient at $Y^{d}$ equal to 1 , it can be decomposed into linear factors

$$
F_{d}\left(x^{\prime}\right)(A, Y)=\left(Y-\xi_{1}\left(x^{\prime}\right) A\right) \ldots\left(Y-\xi_{d}\left(x^{\prime}\right) A\right) .
$$

By Lemma 6.2, there are $m_{1}\left(x^{\prime}\right), \ldots, m_{s\left(x^{\prime}\right)}\left(x^{\prime}\right) \in \mathbb{N}$ such that

$$
B_{V} \subset B_{W\left(x^{\prime}\right)} \subset\left\{k / m_{i}\left(x^{\prime}\right): i=1, \ldots, s\left(x^{\prime}\right), k \in \mathbb{N}\right\}
$$

and

$$
\begin{aligned}
F_{d}\left(x^{\prime}, Y\right) & =\left(Y-\xi_{1}\left(x^{\prime}\right)\right)^{m_{1}\left(x^{\prime}\right)} \ldots\left(Y-\xi_{s\left(x^{\prime}\right)}\left(x^{\prime}\right)\right)^{m_{s\left(x^{\prime}\right)}\left(x^{\prime}\right)} \\
& =\left(Y-\eta_{1}\left(x^{\prime}\right)\right)^{t_{1}\left(x^{\prime}\right)} \ldots\left(Y-\eta_{r\left(x^{\prime}\right)}\left(x^{\prime}\right)\right)^{t_{r\left(x^{\prime}\right)}\left(x^{\prime}\right)},
\end{aligned}
$$

where $\eta_{i}\left(x^{\prime}\right) \neq \eta_{j}\left(x^{\prime}\right)$ for $i \neq j, t_{1}\left(x^{\prime}\right) \leq \ldots \leq t_{r\left(x^{\prime}\right)}\left(x^{\prime}\right), t_{j}\left(x^{\prime}\right)=m_{j, 1}\left(x^{\prime}\right)+$ $\ldots+m_{j, r_{j}\left(x^{\prime}\right)}\left(x^{\prime}\right)$ for $j=1, \ldots, r\left(x^{\prime}\right)$ and $\left\{m_{j, k}\left(x^{\prime}\right): j=1, \ldots, r\left(x^{\prime}\right), k=\right.$ $\left.1, \ldots, r_{j}\left(x^{\prime}\right)\right\}=\left\{m_{i}\left(x^{\prime}\right): i=1, \ldots, s\left(x^{\prime}\right)\right\}$.

Since $\sum_{j=1}^{r\left(x^{\prime}\right)} t_{j}\left(x^{\prime}\right)=d<\infty$, there exists a set $U^{\prime} \subset U$ of the second Baire category such that for all $x^{\prime} \in U^{\prime}$ we have $s\left(x^{\prime}\right)=s, r\left(x^{\prime}\right)=r$, $t_{j}\left(x^{\prime}\right)=t_{j}$ for $j=1, \ldots, r, m_{j, k}\left(x^{\prime}\right)=m_{j, k}$ for $j=1, \ldots, r, k=1, \ldots, r_{j}$.

Let $d_{1}<\ldots<d_{l}$ be natural numbers such that

$$
\begin{gathered}
d_{1}=t_{1}=\ldots=t_{l_{1}}, \quad d_{2}=t_{l_{1}+1}=\ldots=t_{l_{1}+l_{2}}, \ldots, \\
d_{l}=t_{l_{1}+\ldots+l_{l-1}+1}=\ldots=t_{r} .
\end{gathered}
$$

Then for $x^{\prime} \in U^{\prime}$

$$
F_{d}\left(x^{\prime}, Y\right)=\left[q_{1}\left(x^{\prime}\right)(Y)\right]^{d_{1}} \cdot \ldots \cdot\left[q_{l}\left(x^{\prime}\right)(Y)\right]^{d_{l}},
$$

where $q_{i}\left(x^{\prime}\right) \in \mathbb{C}[Y]$ is a monic polynomial of degree $l_{i}$. Moreover, $q_{i}\left(x^{\prime}\right)$ and $q_{j}\left(x^{\prime}\right)$ have no common roots for $i \neq j$, and $q_{i}\left(x^{\prime}\right)$ has no multiple roots.

To complete our proof it suffices to prove the following

Lemma 6.3. Let $U \subset \mathbb{C}^{n}$ be of the second Baire category and let $g \in$ $\mathbb{C}[X, Y]$ be monic on $Y$, where $X=\left(X_{1}, \ldots, X_{n}\right)$. If, for each $x \in U$, $g(x, Y)$ has a root of multiplicity $\geq k$ then $g=p^{k} q$, where $p \in \mathbb{C}[X, Y] \backslash \mathbb{C}$, $q \in \mathbb{C}[X, Y]$ are monic on $Y$.

Indeed, applying Lemma 6.3 to the polynomial $F_{d}\left(X^{\prime}, Y\right)$ and the set $U^{\prime}$ we obtain $F_{d}=p^{d_{l}} q$, with $p \in \mathbb{C}\left[X^{\prime}, Y\right] \backslash \mathbb{C}$ and $q \in \mathbb{C}\left[X^{\prime}, Y\right]$. If $p\left(x^{\prime}\right)$ denotes the polynomial $\left\{y \rightarrow p\left(x^{\prime}, y\right)\right\} \in \mathbb{C}[Y]$ then we have $p\left(x^{\prime}\right) \mid q_{l}\left(x^{\prime}\right)$ for $x^{\prime} \in U^{\prime}$. Thus

$$
q\left(x^{\prime}, Y\right)=\left[q_{1}\left(x^{\prime}\right)(Y)\right]^{d_{1}} \ldots\left[q_{l-1}\left(x^{\prime}\right)(Y)\right]^{d_{l-1}} \cdot\left[q_{l}\left(x^{\prime}\right)(Y) / p\left(x^{\prime}\right)(Y)\right]^{d_{l}} .
$$

Repeated application of Lemma 6.3 to the subsequently obtained remainders $q$ gives us finally $F_{d}=p_{1}^{d_{1}} \ldots p_{l}^{d_{l}}$, where $p_{i} \in \mathbb{C}\left[X^{\prime}, Y\right] \backslash \mathbb{C}, p_{i}\left(x^{\prime}\right)=q_{i}\left(x^{\prime}\right)$ for $x^{\prime} \in U^{\prime}, i=1, \ldots, l$. Hence $\left(p_{i}, p_{j}\right)=1$ for $i \neq j$, and $p_{i}$ has no multiple factors for $i=1, \ldots, l$, which completes the proof of Theorem 6.1. 
Proof of Lemma 6.3. Let $g=p_{1} \ldots p_{s}$ be the decomposition of $g$ in $\mathbb{C}[X, Y]$ into irreducible polynomials monic in $Y$. For $i=1, \ldots, s$ we can treat $p_{i}$ as a monic irreducible polynomial of $Y$ with coefficients in $\mathbb{C}[X]$, so its discriminant $\Delta\left(p_{i}\right) \in \mathbb{C}[X]$ is different from 0 . Hence

$$
W_{0}:=\left\{x \in \mathbb{C}^{n}: \Delta\left(p_{1}\right)(x) \ldots \Delta\left(p_{s}\right)(x)=0\right\}
$$

is a proper algebraic subset of $\mathbb{C}^{n}$. For fixed $x$, let $p_{i}(x)$ denote the polynomial $\left\{y \rightarrow p_{i}(x, y)\right\} \in \mathbb{C}[Y]$. For $x \in U \backslash W_{0}$ there exists a $k$-tuple $\alpha=\left\{\alpha_{1}, \ldots, \alpha_{k}\right\} \subset\{1, \ldots, s\}$ such that $p_{\alpha_{1}}(x), \ldots, p_{\alpha_{k}}(x)$ have a common root, because none of $p_{1}(x), \ldots, p_{s}(x)$ has a multiple root and $q(x)=$ $p_{1}(x) \ldots p_{s}(x)$ has a root of multiplicity $\geq k$. In particular, for any $i, j=$ $1, \ldots, k$, the resultant $R\left(p_{\alpha_{i}}(x), p_{\alpha_{j}}(x)\right)=0$. We have

$$
R\left(p_{\alpha_{i}}(x), p_{\alpha_{j}}(x)\right)=R\left(p_{\alpha_{i}}, p_{\alpha_{j}}\right)(x),
$$

where $R\left(p_{\alpha_{i}}, p_{\alpha_{j}}\right) \in \mathbb{C}[X]$ is the resultant of $p_{\alpha_{i}}, p_{\alpha_{j}}$ as polynomials of $Y$. Hence the sets

$$
W_{\alpha}:=\left\{x \in \mathbb{C}^{n}: R\left(p_{\alpha_{i}}(x), p_{\alpha_{j}}(x)\right)=0 \text { for } i, j=1, \ldots, k\right\},
$$

where $\alpha=\left(\alpha_{1}, \ldots, \alpha_{k}\right) \subset\{1, \ldots, s\}$, are algebraic subsets of $\mathbb{C}^{n}$. Since $\bigcup_{\alpha \subset\{1, \ldots, s\}} W_{\alpha} \supset U \backslash W_{0}$ is of the second Baire category, there exists $\alpha$ such that $W_{\alpha}=\mathbb{C}^{n}$. Thus $R\left(p_{\alpha_{i}}(x), p_{\alpha_{j}}(x)\right)=0$ for $i, j=1, \ldots, k$ and $x \in \mathbb{C}^{n}$. Hence $R\left(p_{\alpha_{i}}, p_{\alpha_{j}}\right)=0$ in $\mathbb{C}[X]$. Therefore $p_{\alpha_{i}}, p_{\alpha_{j}}$ have a common root $\xi$ in the algebraic closure of $\mathbb{C}(X)$, and so are both divisible by the minimal polynomial of $\xi$ in $\mathbb{C}(X)[Y]$. However, being monic and irreducible in $\mathbb{C}[X][Y]$, they are irreducible in $\mathbb{C}(X)[Y]$. Hence $p_{\alpha_{i}}=p_{\alpha_{j}}=: p$ for $i, j=1, \ldots, k$.

\section{References}

[1] R. Draper, Intersection theory in algebraic geometry, Math. Ann. 180 (1969), 19752040.

[2] S. Łojasiewicz, Introduction to Complex Analytic Geometry, Birkhäuser, to appear in 1991.

[3] D. Mumford, Algebraic Geometry, Vol. 1, Complex Projective Varieties, Springer, Berlin 1976.

[4] P. Tworzewski and T. Winiarski, Analytic sets with proper projections, J. Reine Angew. Math. 337 (1982), 68-76.

[5] T. Winiarski, Continuity of total number of intersection, Ann. Polon. Math. 47 (1986), 155-178.

INSTITUTE OF MATHEMATICS

JAGIELLONIAN UNIVERSITY

REYMONTA 4

30-059 KRAKÓW, POLAND 